\title{
Diversification of MIF immune regulators in aphids: link with agonistic and antagonistic interactions
}

Géraldine Dubreuil ${ }^{1,2}$, Emeline Deleury ${ }^{1}$, Didier Crochard ${ }^{1}$, Jean-Christophe Simon ${ }^{3}$ and Christine Coustau ${ }^{*}$

\begin{abstract}
Background: The widespread use of genome sequencing provided evidences for the high degree of conservation in innate immunity signalling pathways across animal phyla. However, the functioning and evolutionary history of immune-related genes remains unknown for most invertebrate species. A striking observation coming from the analysis of the pea aphid Acyrthosiphon pisum genome is the absence of important conserved genes known to be involved in the antimicrobial responses of other insects. This reduction in antibacterial immune defences is thought to be related to their long-term association with beneficial symbiotic bacteria and to facilitate symbiont maintenance. An additional possibility to avoid elimination of mutualistic symbionts is a fine-tuning of the host immune response. To explore this hypothesis we investigated the existence and potential involvement of immune regulators in aphid agonistic and antagonistic interactions.

Results: In contrast to the limited antibacterial arsenal, we showed that the pea aphid Acyrthosiphon pisum expresses 5 members of Macrophage Migration Inhibitory Factors (ApMIF), known to be key regulators of the innate immune response. In silico searches for MIF members in insect genomes followed by phylogenetic reconstruction suggest that evolution of MIF genes in hemipteran species has been shaped both by differential losses and serial duplications, raising the question of the functional importance of these genes in aphid immune responses. Expression analyses of ApMIFs revealed reduced expression levels in the presence, or during the establishment of secondary symbionts. By contrast, ApMIFs expression levels significantly increased upon challenge with a parasitoid or a Gram-negative bacteria. This increased expression in the presence of a pathogen/parasitoid was reduced or missing, in the presence of facultative symbiotic bacteria.
\end{abstract}

Conclusions: This work provides evidence that while aphid's antibacterial arsenal is reduced, other immune genes widely absent from insect genomes are present, diversified and differentially regulated during antagonistic or agonistic interactions.

Keywords: Invertebrate immunity, Cytokines, Macrophage migration inhibitory factor, Host-parasite interactions, Host-symbiont interactions, Acyrthosiphon pisum

\footnotetext{
* Correspondence: Christine.coustau@sophia.inra.fr

'Sophia Agrobiotech Institute, INRA-CNRS-UNS, UMR 7254, 400 Route des

Chappes, 06903 Sophia Antipolis, France

Full list of author information is available at the end of the article
} 


\section{Background}

Invertebrate immune systems have been shown to include highly diversified recognition systems, complex regulatory processes, and specific effectors [1-5]. However, the functioning and evolutionary history of immune-related genes remains unknown for most invertebrate species.

For example, the recent sequencing of the first aphid genome (Acyrthosiphon pisum) provided new insights into insect immunity but also raised novel evolutionarily- and functionally-intriguing questions [6]. Indeed, genes participating in the immune responses of other insects are missing in aphids, such as the microbial recognition genes PGRPs (peptidoglycan receptor proteins) involved in the recognition of both Gram-negative and -positive bacteria $[7,8]$. Even more intriguing is the absence of most of the components of the immunodeficiency (IMD) signaling pathway, which is critical for fighting Gram-negative bacteria, and the absence of most of the antimicrobial peptides conserved in other insects $[7,8]$. These results, further supported by expression studies [8], showed that the aphid immune system greatly differs from that of other well studied insects and it was hypothesized that aphids compensate for a deficient immune system by symbiont-mediated host protection and an extraordinary reproduction rate [8]. Aphids are plant sap-feeding insects associated with the obligatory nutrient-providing symbiont Buchnera aphidicola $[9,10]$. Aphids also interact with several species of facultative bacterial endosymbionts [11] that are found free in the hemolymph $[11,12]$. Interestingly, secondary symbionts have been shown to influence important fitness-related traits such as body pigmentation [13], offspring production [14] and resistance to parasitoids [15-17] or pathogens [18]. It has been hypothesized that these intimate aphid-symbiont interactions may have led to the loss of specific antibacterial compounds or pathways to accommodate long-term host-symbiont coevolution and symbiont maintenance [19].

An additional possibility to avoid elimination of mutualistic symbionts is a fine-tuning of the host immune response [20,21]. Aphid immune system may be particularly well regulated to prevent or limit damage to their bacterial mutualists under pathogen infections. As a first approach to explore this hypothesis, we searched $A$. pisum genome for immune regulators, and we noticed the existence of five genes coding for Macrophage Migration Inhibitory factors (MIFs) within the A. pisum genome. In vertebrates, MIFs are important pro-inflammatory cytokines acting on key cellular processes of the immune response such as cell proliferation and apoptosis [22,23]. MIFs were identified in a variety of species, including protozoan, nematode, mollusk and crustacean species [5,24-29]. It was shown that a mollusk MIF (BgMIF1) not only presented the expected activities on cell proliferation and apoptosis but played a major role in the response against parasitic infection [5]. This study provided functional evidence of the conservation of major immune-related functions of MIFs in an invertebrate and highlighted the importance of these immune regulators in invertebrate immunity [5]. Interestingly, all species investigated so far presented one or two MIF gene copies. The existence of additional MIF copies in $A$. pisum therefore requires dedicated evolutionary and functional analyses to better understand the complex immune interactions of aphids with their symbionts and pathogens.

Here we characterized $A$. pisum MIF members and explored their existence and evolutionary history in various insect phyla. To gain insights into their functional role, we analyzed the expression of the several MIF copies during aphid-symbiont-pathogen interactions.

\section{Results}

\section{Acyrthosiphon pisum MIF (ApMIF) family members}

The search for predicted MIF gene sequences in the annotated genome of Acyrthosiphon pisum [6] (Acyr_2.0 assembly) revealed 5 MIF members referred to as ApMIF1 to ApMIF5 (Table 1). Using BLAST searches [30], we found one or several full-length ESTs for each ApMIF member supporting their existence, structural annotation and expression. Complementary searches evidenced an additional hit, corresponding to a 6th MIF gene that was actually annotated in a previous version of the genome assembly (Acyr_1.0) (Table 1). BLAST searches using the originally predicted ApMIF6 cDNA sequence against the 214920 redundant ESTs resulted in a

Table 1 Accession numbers and summary information on ApMIF genes and their products

\begin{tabular}{|c|c|c|c|c|c|c|}
\hline & $\begin{array}{c}\text { Aphidbase } \\
\text { Genes }\end{array}$ & $\begin{array}{l}\text { NCBI } \\
\text { Genes }\end{array}$ & $\begin{array}{c}\text { NCBI } \\
\text { RefSeq mRNA }\end{array}$ & $\begin{array}{c}\text { NCBI } \\
\text { RefSeq proteins }\end{array}$ & $\begin{array}{c}\text { PF01187 } \\
\text { e-value }\end{array}$ & $\begin{array}{c}\text { Exp. } \\
\text { validation }\end{array}$ \\
\hline$\overline{A p M I F 1}$ & ACYPI002465 & LOC100161225 & NM_001162635.1 & NP_001156107.1 & $1.53 e-38$ & Yes \\
\hline ApMIF2 & ACYPI006088 & LOC100165124 & XM_001946905.2 & XP_001946940.1 & $5.85 e-24$ & Yes \\
\hline ApMIF3 & ACYPI000036 & LOC100144890 & NM_001126157.2 & NP_001119629.1 & $4.61 e-25$ & Yes \\
\hline ApMIF4 & ACYPI003547 & LOC100162394 & NM_001162060.1 & NP_001155532.1 & $2.51 e-24$ & Yes \\
\hline ApMIF5 & ACYPI005907 & LOC100164929 & XM_001948047.2 & XP_001948082.1 & $6.43 e-15$ & Yes \\
\hline ApMIF6 & ACYPI002954 & LOC100161756 & Withdrawn & Withdrawn & - & No \\
\hline
\end{tabular}

Experimental validation (Exp. validation) consisted in the amplification and resequencing of cDNAs. 
partial and fragmented alignment with only 4 similar ESTs that did not support the existence of a full length transcript from a 6th MIF gene. In order to further characterize ApMIF members, RT-PCR amplification of the complete coding sequences of the predicted ApMIF genes was performed from RNA of two genetically different lineages of A. pisum (LL01 and YR2). Amplicons were obtained and resequenced for ApMIF1 to -5. Sequence results confirmed the expression of full length transcripts of ApMIF1 to ApMIF5. Amplification of a full length cDNA from ApMIF6 (based on originally predicted sequence) was however not obtained.

The five predicted ApMIF proteins showed highly significant hits with Macrophage Migration Inhibitory factor domain (PF01187/IPR001398) (Table 1). Interestingly, sequences of the 5 ApMIF predicted proteins differ considerably among each other (Additional file 1: Figure S1) and present $33 \%$ to $55 \%$ similarity in their sequences. MIF proteins from mammals and snails have been shown to catalyze the ketoenol isomerization of small aromatic substrates such as hydroxyphenylpyruvate and L-dopachrome methyl ester [5,31]. Post-translational cleavage of the initiating methionine exposes an $\mathrm{N}$-terminal catalytic proline (Pro2) that is essential for MIF tautomerase activity [32]. Three of the five predicted ApMIF proteins present this Proline2 residue as well as other conserved residues such as Lys33, Ile65 and Tyr93 and Val107, participating to MIF active sites [33] (see Additional file 1: Figure S1 for alignment of ApMIF sequences). Another conserved feature of MIF proteins is to be secreted via non-classical pathways, involving, for example an ATP binding cassette transporter [34]. As expected, no signal peptides were detected in ApMIF sequences (SignalP 4.0; [35]) but the
SecretomeP server [36] predicted ApMIF 1 and ApMIF 2 as secreted proteins (secP score 0.934 and 0.78 respectively).

\section{MIF family members in insects}

In order to further characterize this multigenic family, we first searched for $M I F$ genes in sequenced insect genomes. Intriguingly, with the exception of two MIF genes in Tribolium castaneum and in Bombyx mori, no MIF genes were identified from the other insect genomes available at the time of the study (Table 2). We then performed an extensive search for MIF transcripts from 27 hemipteran species (Table 3) with available ESTs in public databases. Interestingly, MIF transcripts were detected, in particular, in phloem-sap feeding species belonging to the Delphacidae, Aphididae, Pemphigidae, Pseudococcidae and Psyllidae families (Table 3). MIF genes were identified in all Aphididae with available ESTs with up to 5 members. Note that except for A. pisum the number of MIF members is probably underestimated due to the absence of annotated genomic data and to the modest size of some of the transcriptomic databases. Aphid genomic and transcriptomic data are currently growing rapidly and will allow a complete identification of aphid MIF members in a close future.

\section{Phylogenic reconstruction of MIFs from hemipterans}

Phylogenic reconstruction of complete predicted MIF sequences from hemipterans was performed using the crustacean Daphnia pulex as an outgroup (Figure 1). With the exception of A. pisum ApMIF5, all predicted MIF proteins from Delphacidae and Aphididae clustered into five clades including one clade specific of Delphacidae and 4 clades specific of Aphididae (Figure 1). Each

Table 2 Survey of the number of MIF genes in insect genomes

\begin{tabular}{lll}
\hline & Species & Nb MIF \\
\hline Diptera & Drosophila species (r5.46, ftp://ftp.flybase.org) & 0 \\
& Anopheles gambiae (r3.6, ftp://ftp.flybase.org) & 0 \\
Lepidoptera & Aedes aegypti (r1.3, http://aaegypti.vectorbase.org/) & 0 \\
Coleoptera & Bombyx mori (http://www.silkdb.org/) & 2 \\
Hymenoptera & Tribolium castaneum (v2.0, http://www.hgsc.bcm.tmc.edu/) & 2 \\
& Acromyrmex echinatior (v3.8, http://www.antgenomes.org/) & 0 \\
& Apis melifera (r4.5, http://hymenopteragenome.org/beebase/) & 0 \\
Apis florea (v1.0, http://www.hgsc.bcm.tmc.edu/) & 0 \\
Hemiptera & Bombus impatiens (v2.0, http://hymenopteragenome.org/beebase/?q=gbrowse_bimp) \\
Cladocera & Bombus terrestris (v1.1, http://www.hgsc.bcm.tmc.edu/) & 0 \\
Nasonia vitripennis (v0.5, http://www.hgsc.bcm.tmc.edu/) & Acyrthosiphon pisum (v2.1, http://www.aphidbase.com) & 0 \\
\hline Bediculus humanus (u1.2, https://www.vectorbase.org/) & 0 \\
\hline
\end{tabular}

Between parentheses are indicated the assembly version numbers and the URL used for genomic database searches. Daphnia pulex is added as an outgroup. 
Table 3 Survey of complete MIF family members from 27 available hemiptera species

\begin{tabular}{|c|c|c|c|c|c|}
\hline Order & Suborder & Family & Species & Num EST & $\mathrm{Nb}$ MIF \\
\hline \multirow[t]{27}{*}{ Hemiptera } & Auchenorryncha & Cicadellidae & Graphocephala atropunctata & 6481 & 0 \\
\hline & & & Homalodisca vitripennis & 20030 & 0 \\
\hline & & & Oncometopia nigricans & 9056 & 0 \\
\hline & Heteroptera & Delphacidae & Nilaparvata lugens (NI) & 118020 & 2 \\
\hline & & & Peregrinus maidis (Pm) & 20678 & 2 \\
\hline & & Cimicidae & Cimex lectularius & 7129 & 0 \\
\hline & & Miridae & Adelphocoris lineolatus & 2915 & 0 \\
\hline & & Reduviidae & Dipetalogaster maximus & 2671 & 0 \\
\hline & & & Rhodnius prolixus & 16105 & 0 \\
\hline & & & Triatoma brasiliensis & 2115 & 0 \\
\hline & & & Triatoma infestans & 2564 & 0 \\
\hline & & & Triatoma matogrossensis & 2230 & 0 \\
\hline & & & Triatoma rubida & 1850 & 0 \\
\hline & & Lygaeidae & Oncopeltus fasciatus & 1115 & 0 \\
\hline & Sternorrhyncha & Aleyrodidae & Bemisia tabaci & 11923 & 0 \\
\hline & & Aphididae & Aphis gossypii (Ag) & 88851 & 2 \\
\hline & & & Rhopalosiphum maidis (Rm) & 17649 & 4 \\
\hline & & & Rhopalosiphum padi (Rp) & 17892 & 3 \\
\hline & & & Toxoptera citricida (Tc) & 4304 & 2 \\
\hline & & & Acyrthosiphon kondoi (Ak) & 23040 & 2 \\
\hline & & & Acyrthosiphon pisum (Ap) & 214834 & 5 \\
\hline & & & Myzus ascalonicus (Ma) & 22942 & 5 \\
\hline & & & Myzus persicae (Mp) & 27728 & 3 \\
\hline & & & Sitobion avenae (Sa) & 3478 & 1 \\
\hline & & Pemphigidae & Pemphigus spyrothecae & 18587 & 0 \\
\hline & & Pseudococcidae & Maconellicoccus hirsutus (Mh) & 7669 & 2 \\
\hline & & Psyllidae & Diaphorina citri (Dc) & 19598 & 1 \\
\hline
\end{tabular}

The total number of redundant ESTs (Num EST) available in public databases at the time of the study is shown for each species, as well as the number of complete cDNA unique sequences predicted to code MIF proteins (presence of PF01187 domain confirmed by pfam_scan). Between parentheses are shown the species identification letters as used for the phylogenetic reconstruction.

ApMIF member clustered into one of the four clades, together with MIF members from other aphid species (Figure 1), showing that several duplications occurred before the diversification of aphids but after the split of Aphididae from the other hemipterans.

\section{ApMIFs constitutive expression}

Expression of the $5 A p M I F$ transcripts was analysed by real time RT-PCR on 12-day-old aphids from YR2 and LL01 free of any secondary symbionts [14,37]. In both lineages, transcripts of ApMIF1, ApMIF2 and ApMIF3, respectively clustered in clade $\mathrm{A}, \mathrm{C}$ and $\mathrm{D}$, were highly detected in whole bodies while ApMIF4 and ApMIF5 showed very weak expression levels (Figure 2). All five ApMIFs transcripts were detected in hemocytes, supporting their potential involvement in immune processes (Figure 2B). To better assess the presence of ApMIFs in hemocytes, we used an antibody raised against peptides of one of the well-expressed ApMIFs (ApMIF1). As shown in the Figure 2C, ApMIF1 protein localizes within hemocyte granules.

Further studies on ApMIF expression were carried out on the 5 ApMIFs. However, because expression levels of ApMIF4 and ApMIF5 remained at the limit of detection by real time quantitative RT-PCR in each experiment, they are not presented in the following result sections.

\section{Increased ApMIF expression after immune challenges}

To explore the expected involvement of ApMIF genes in aphid immune response, we examined their expression levels after immune challenges. We first used Aphidius ervi, which is the main parasitoid of $A$. pisum in the field [38]. The two clones of $A$. pisum showed a significant increase in ApMIF1, ApMIF2 and ApMIF3 transcript levels 


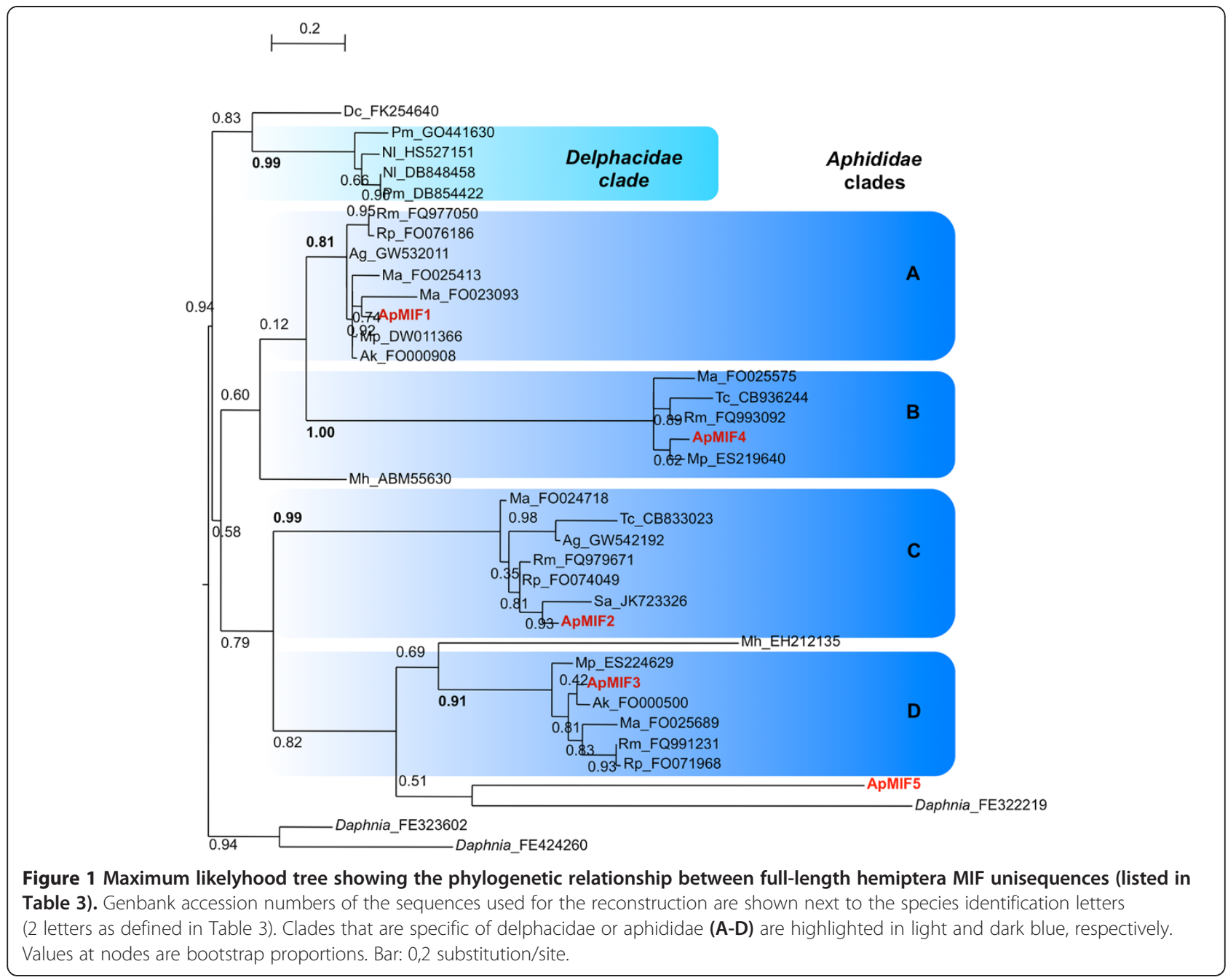

after exposure to female parasitoid (Figure 3). An increased ApMIF expression is also observed in aphids after injection with the Gram-negative bacteria E. coli (Figure 3).

\section{Decreased ApMIF expression during interactions with mutualistic symbionts}

We first compared the expression levels of ApMIF transcripts in the three lineages derived from YR2 and each harbouring a specific secondary symbiont (YR2-Hd, YR2$\mathrm{Ri}$ and YR2-Ss). Aphids carrying mutualistic symbionts showed lower expression levels of ApMIF1, ApMIF2 and ApMIF3 (Figure 4). In order to further study changes in ApMIF expression in presence of secondary symbionts, we measured ApMIF expression levels in LL01 following the injection of each of the three secondary symbionts. Since it was reported that injection of hemolymph from infected to uninfected A. pisum can establish a stable infection of secondary facultative symbionts [39,40], each secondary symbiont was manually collected from YR2 lineages and injected into LL01 adults. ApMIF expression was measured 1 and 4 days after injection. ApMIF1,
ApMIF2 and ApMIF3 expression levels rapidly decreased after infection as compared to controls injected with Schneider medium (Figure 5). To ascertain that experimental infections with the facultative symbionts were successful, individuals from the F1 progeny of injected adults were microscopically examined. Presence of bacteria in the hemolymph confirmed the establishment of these vertically transmitted symbionts, and analyses of ApMIFs expression levels confirmed that their decreased expression persisted in the F1 progeny (Figure 5). Altogether, these results showed that the establishment and the presence of the three major facultative symbionts of $A$. pisum correlate with a decreased expression of the three well-expressed ApMIF genes.

\section{ApMIF expression during both agonistic and antagonistic interactions}

Because some secondary symbionts can impact important aphid fitness-related traits such as resistance to parasitoids [15-17] or pathogens [18], it may be disadvantageous for aphids to mount a complete immune response in 

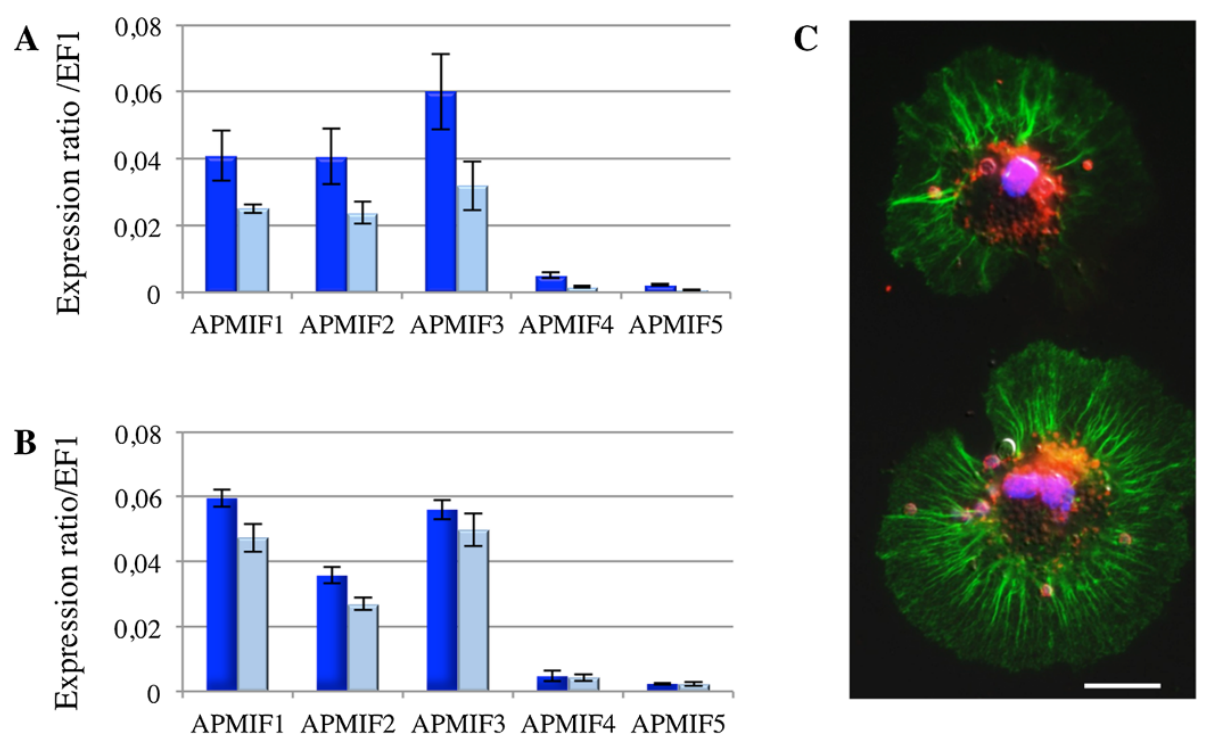

Figure 2 Expression ratios (normalized to EF1 expression) of the five ApMIF transcripts in whole bodies (A) and hemocytes (B) from the $\boldsymbol{A}$. pisum genetic lineages LL01 (dark blue) and YR2 (light blue). Each bar represents the mean expression ratio \pm SD obtained from three independent experiments. (C) Immunolocalization of ApMIF1 in hemocytes. Merger of fluorescent micrographs showing two hemocytes with labeled actin (green fluorescence), nuclei (purple/bleu fluorescence) and ApMIF1 protein (orange fluorescence). Scale bar represents $10 \mu \mathrm{m}$.

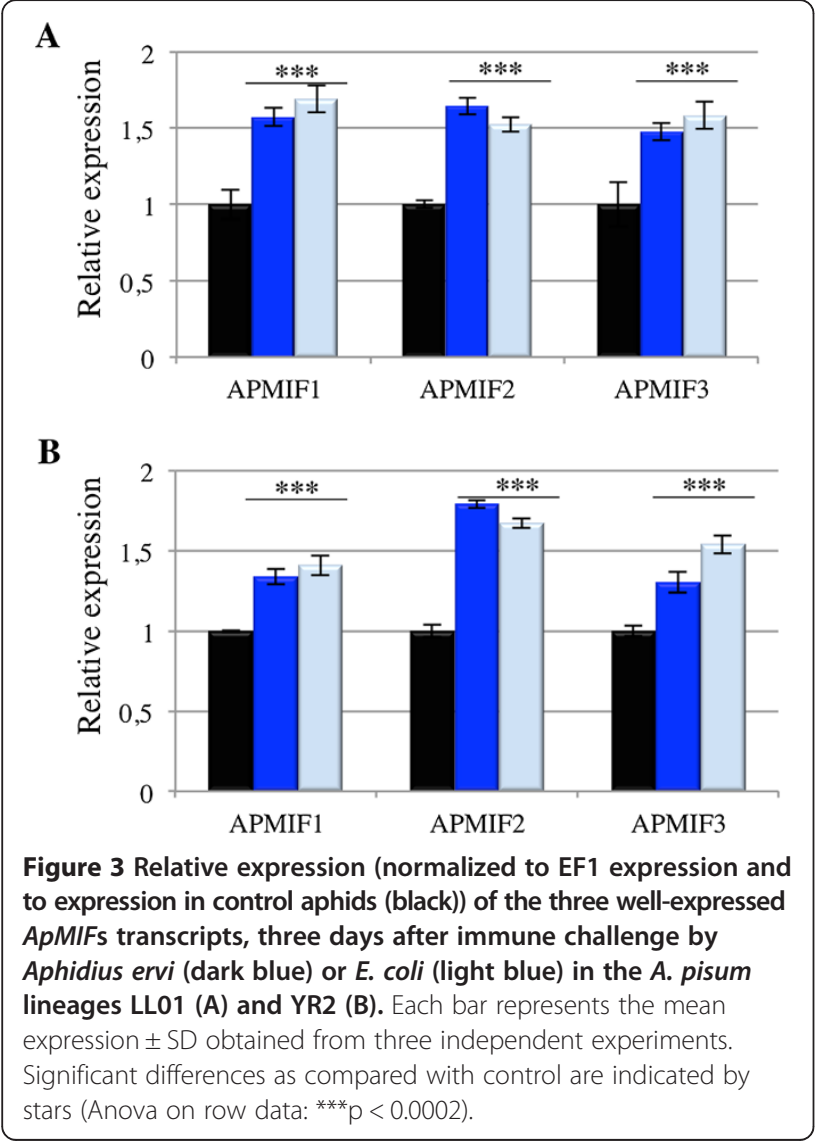

the presence of both these mutualists and a pathogen or parasitoid. Similarly, if the negative regulation of aphid immune response results from a direct effect of mutualists on host immune system, one may expect that this regulation would be maintained after an immune challenge and would limit the increase in ApMIF expression. To test this hypothesis, ApMIF expression levels were analyzed after exposure to parasitoids in the YR2 lineages carrying each of the three facultative symbionts. No significant change in gene expression was observed for ApMIF1 (Figure 6A). Increased expressions were observed for ApMIF2 and ApMIF3 after exposure to the parasitoid A. ervi (Figure 6B,C), the highest expression levels being observed in the YR2-Ss lineage. However, all expression levels remained far below those of the YR2 lineage without mutualistic symbionts (Figure 6).

\section{Discussion}

Widespread lineage-specific gene duplications have been observed in the A. pisum genome [6]. The number of duplications in this aphid appears greater than that of any other sequenced insect with over 2000 gene families that have undergone gene duplication [6]. However, these duplications were not reported to particularly affect immune-related genes, and, on the contrary, important and conserved genes of the antibacterial pathways are missing from $A$. pisum genome [6].

A specific search for MIF genes in genomes from insects revealed that except for Tribolium castaneum and Bombyx mori, no other genome contained MIF members. 


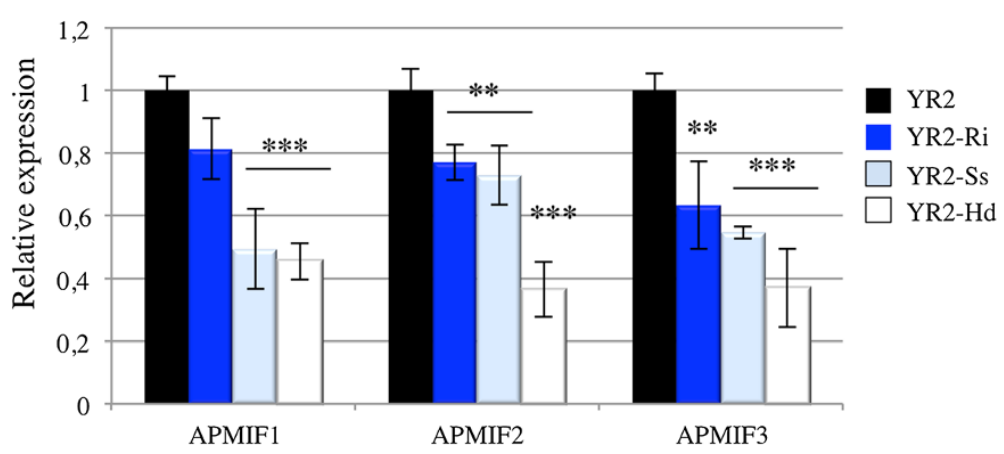

Figure 4 Comparison of ApMIFs expression in YR2 aphids associated with R. insecticola (YR2-Ri), S. symbiotica (YR2-Ss) or H. defensa (YR2-Hd). Expression has been normalized to EF1 expression and to expression in control aphids (black). Each bar represents the mean expression \pm SD obtained from three independent experiments. Significant differences as compared with control are indicated by stars (Anova on row data: ***: $p<0.0002 ;{ }^{* *}: p<0.002$ ).

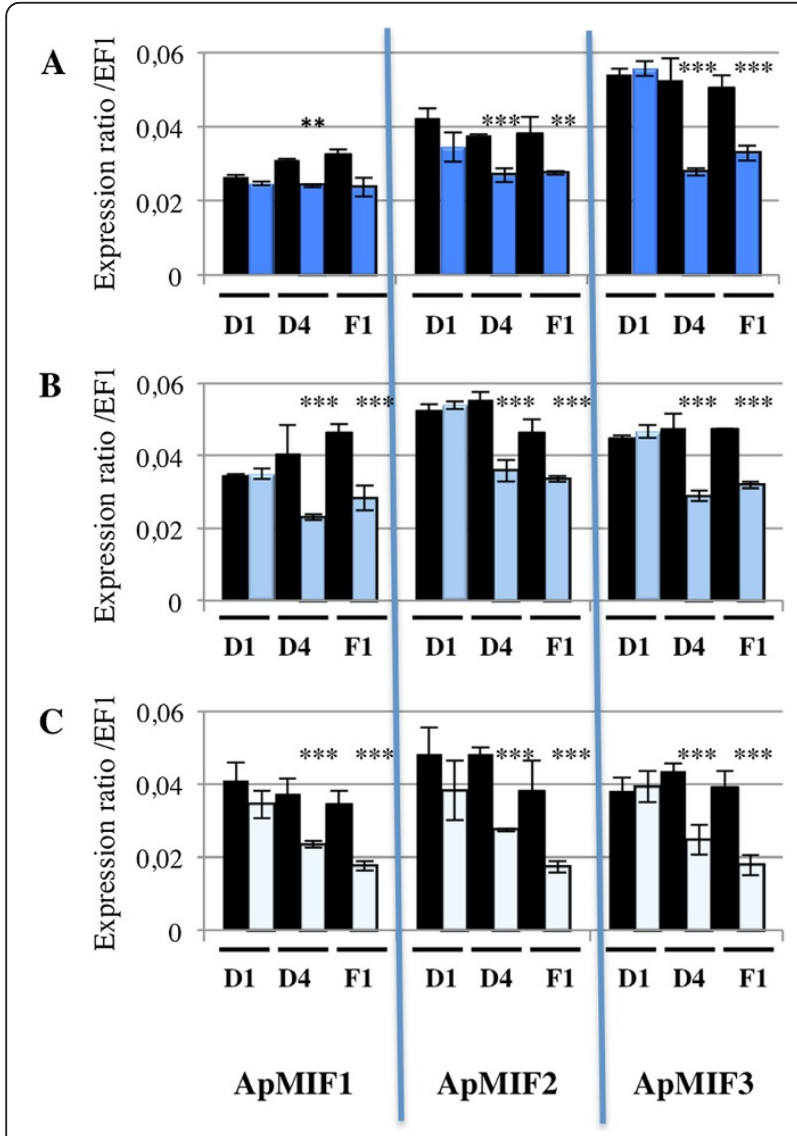

Figure 5 Effect of secondary symbiont injection on ApMIFs expression in LL01 aphids injected with $R$. insecticola (A), $S$. symbiotica (B) or $H$. defensa (C). Expression was measured at one (D1) or four (D4) days post injection, as well as in offspring from the first generation (F1), in symbiont injected (blue) and buffer injected (black) aphids. Significant differences as compared with control are indicated by stars (Anova on row data: ***: $p<0.0002$; ${ }^{* *}$ p $<0.002$ ).
Conversely, analysis of hemipteran transcriptomic databases provided evidence for the existence of a MIF multigenic family in Aphididae species as well as for the existence of at least 2 MIF members in Delphacidae species. Phylogenic reconstruction of complete predicted MIF sequences from hemipterans showed that sequences clustered into five clades including one clade specific of Delphacidae and 4 clades specific of Aphididae. Each Aphididae clade contained closely related MIF members from several aphid species showing that duplications occurred before the diversification of aphids. Altogether, these results showed that losses occurred in most insect phyla while both differential losses and duplications occurred within the hemiptera phylum. This differential loss versus duplication, together with the previously observed absence of important immune-related genes in A. pisum genome, raised the question of the functional significance of MIF members in Aphididae.

Detection of the ApMIF transcripts in aphid whole bodies and hemocytes is consistent with the expected expression of these cytokines. Mammalian MIFs are expressed in various cell types, including all immunerelevant tissues (e.g. lung and digestive epithelia, skin) and macrophages [22]. In mammals, MIF genes are constitutively expressed and the protein is stored in granules of immune-relevant cells and rapidly secreted in extracellular fluids upon challenge $[41,42]$. Here we showed that ApMIF proteins are also detected in immune cell granules, suggesting that they are stored and secreted upon challenge. In addition to the expression localization, the significant increase in ApMIF expression after immune challenges support the role of these genes in promoting and regulating major early processes of the innate immune response [22]. MIFs are pleiotropic proteins involved in numerous cellular processes and are key cytokines controlling the response to both parasitic and bacterial infections [5,22,43,44]. The over-expression of ApMIFs was observed in two distinct aphid clones and 

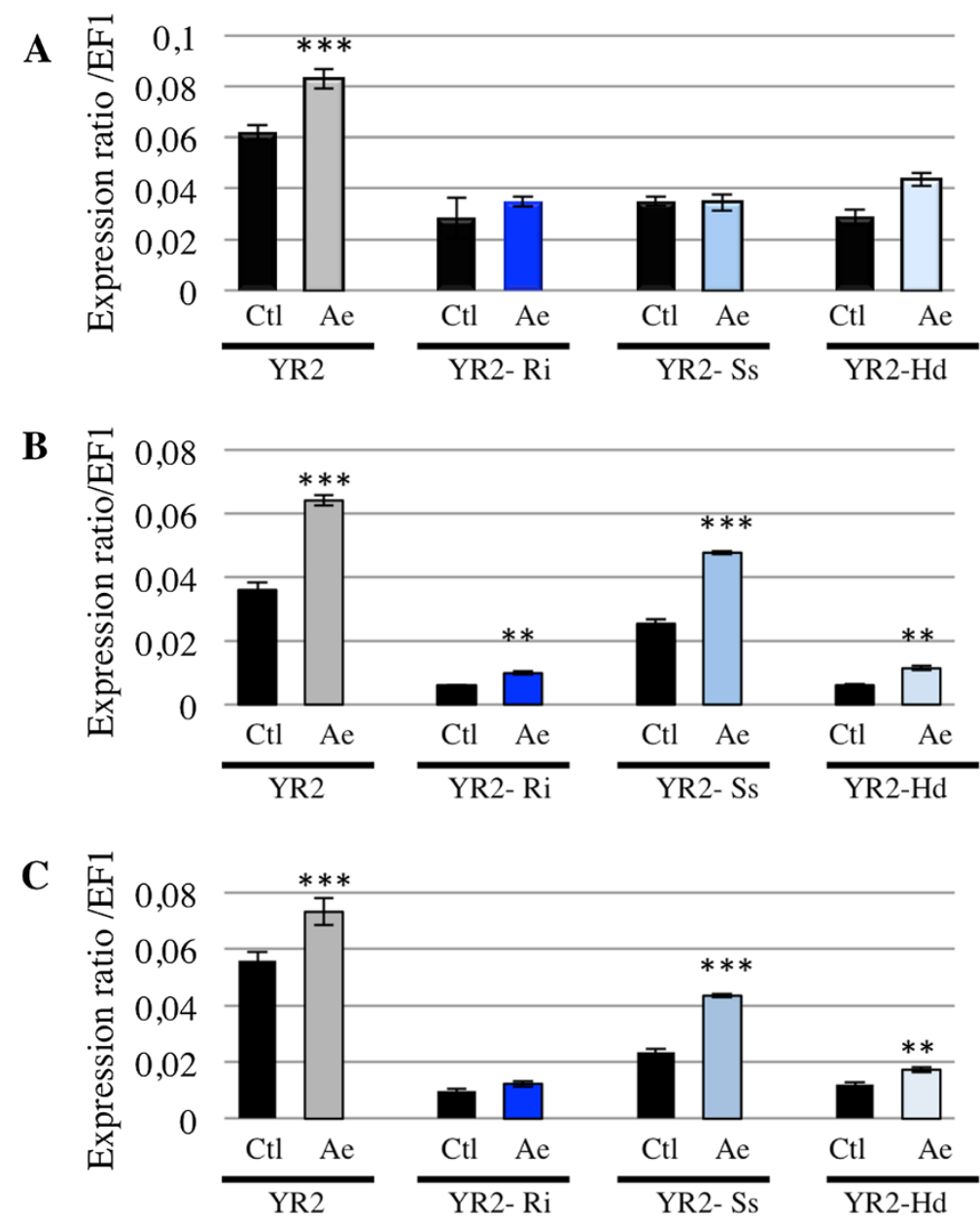

Figure 6 Expression levels of ApMIF1 (A), ApMIF2 (B) and ApMIF3 (C) in the YR2 lineage without symbiont (YR2) or associated with R. insecticola (YR2-Ri), S. symbiotica (YR2-Ss) or H. defensa (YR2-Hd), at 3 days post-exposure to the parasitoid A. ervi (Ae). Significant differences in expression as compared with control aphids (ctl) not exposed to A. ervi are indicated by stars (Anova on row data: ${ }^{* * *} \mathrm{p}<0.0002$; **: $p<0.002)$.

after exposure to both parasitoids and pathogenic bacteria, supporting a general involvement of ApMIFs in aphid's immune response, regardless of the aphid genetic background or of the pathogen.

Interestingly, a lower expression of ApMIF genes was observed either constitutively or after immune challenge in aphid lineages infected by one of the three tested secondary symbionts ( $R$. insecticola, S. symbiotica and $H$. defensa). A recent study showed that the number of $A$. pisum hemocytes is significantly smaller in YR2 lineages carrying these secondary symbionts as compared with the YR2 lineage without secondary symbionts [12]. The decreased expression levels of MIF genes that we observed in these YR2 lineages may therefore result partly from their smaller hemocyte number and partly from a down regulation of expression.
These results are consistent with previous observations on the association between the weevil Sitophilus and the Sitophilus primary endosymbiont (SPE) [45]. In contrast to aphids, weevil immune system presents several families of conserved antimicrobial peptides [45] and it was shown that the antimicrobial peptide coleoptericin-A (ColA) inhibits bacterial cell division and is essential in the regulation of endosymbiont number and location [19]. In addition, although expression of antimicrobial peptides (AMP) was similar in symbiotic (carrying SPE) and aposymbiotic larvae, a lower AMP gene expression was observed after immune challenge with E. coli in symbiotic insects [45]. Here, we observed a negative effect of symbionts on expression of another type of immune genes in aphids, further supporting the importance of immune gene expression in regulating both agonistic and antagonistic interactions. 


\section{Conclusions}

The present study shows that members of the Macrophage Migration Inhibitory Factor (MIF) present a complex evolutionary history characterized by differential losses and duplications across insect phyla.

The 5 MIF members of A. pisum are functional, expressed in circulating immune cells and differently regulated during a pathogenic or mutualistic interaction.

This work provides evidence that while aphid's antibacterial arsenal is reduced, immune regulators widely absent from insect genomes are present, diversified and differentially regulated during antagonistic or agonistic interactions. This supports the hypothesis of a fine-tuning of the immune response possibly accommodating both symbiont maintenance and response to aggressors.

\section{Methods}

\section{Database searches and sequence analyses}

Protein sequences encoded by MIF genes in A. pisum genome were identified using BLAST programs [30] at the Aphidbase (available at http://www.aphidbase.com/) and National Centre for Biotechnology Information (NCBI) servers, providing a 6.2X-coverage genome assembly (Acyr_2.0 and Acyr_1.0) of A. pisum [6]. Other public databases used included the Reference Sequence (RefSeq) database [46] for A. pisum mRNA and protein sequences and the 214,920 A. pisum ESTs available at the time of the study in NCBI-dbEST.

An exhaustive search of Hemiptera MIF transcripts in the NCBI's databases (NR, dbEST) was performed using the BLAST program [30]. The predicted translation of each full-length or partial sequence was checked for the presence of the conserved MIF domain (PFAM reference number PF01187 and/or InterPro reference number IPR001398) using pfam_scan software (available at http://pfam.sanger.ac.uk/search). Quality of sequences and their annotations was validated by multiple alignments using Muscle [47]. Only full-length proteins were then used, re-aligned with Muscle and cleaned using Gblocks (version 0.91b, available at http://www.phylogeny.fr). A maximum likelihood phylogenetic tree was generated using PHYML sofware (version 3) [48] and the WAG model of amino acids subsititution matrice. Bootstrap values were obtained from 1000 replicate samples using the proportion of invariable sites and gamma distribution parameters defined by PHYML.

\section{Aphid lineages}

Two distinct clones of A. pisum were used in this study, LL01 [37] and YR2 [14], both carrying only the obligate symbiont Buchnera aphidicola. For YR2, we also used 3 different lineages derived from the same genetic background but each differing by their respective secondary symbionts, being Regiella insecticola (U-type or PAUSS),
Hamiltonella defensa (T-type or PABS), or Serratia symbiotica (R-type or PASS) [14]. These lineages are referred to as YR2-Ri, YR2-Hd and YR2-Ss, respectively.

Aphids were reared on Vicia faba under long photoperiod (16 hours light/8 hours dark) and temperature $\left(19^{\circ} \mathrm{C}\right)$ conditions to maintain continuous parthenogenetic reproduction [49]. Parthenogenetic wingless adults (1215 days old) were used for all experiments except for parasitism by Aphidius ervi that was carried on 3 daysold larvae.

\section{Hemocyte collection}

Hemolymph was collected in a total volume of $200 \mu \mathrm{L}$ of aphid Schneider medium (Sigma-Aldrich), as previously described [12]. Hemolymph was centrifuged at $200 \mathrm{~g}$ for 5 minutes to eliminate putative embryos and hemocytecontaining supernatant was either used for total RNA extraction or further processed for immunolabeling.

\section{Immunolabeling of ApMIF1 in A.pisum hemocytes}

Hemocytes were fixed in paraformaldehyde $4 \%$ in phosphate buffer $0.1 \mathrm{M}$ pH7.2 for 10 minutes and washed 3 times in PBS under agitation. Cells were then permeabilized with Triton $\mathrm{X} 100$ at $0.1 \%$ in PBS for 10 minutes and washed 3 times with PBS under agitation. Hemocytes were incubated with a custom-made antibody raised against two ApMIF1 peptides (shown in the Additional file 1: Figure S1) (diluted 1/1000 in PBS1X-0.05\% tritonX100) for 1 hour at room temperature. Control hemocytes were incubated with rabbit pre-immune serum (diluted $1 / 1000$ in PBS1X-0.05\% Triton X100). After three washes in PBS, hemocytes were incubated for 1 hour at room temperature in anti-rabbit Alexa Fluor 488 secondary antibody (diluted 1/500 in PBS1X-0.05\% Triton X100) and anti-phalloidin-X5-FluoProbe (diluted 1/200 in PBS-0.05\% Triton X100) to detect actin cytoskeleton. After 3 washes in PBS, nucleuses were marked by incubation in DAPI $(1 \mu \mathrm{g} / \mathrm{mL})$ for 10 minutes. Hemocytes were mounted with anti-fading agent (Roti ${ }^{\circ}$-Mount Fluorcare, Carl Roth) and observed with a microscope Axioplan2 (Carl Zeiss) equipped for epifluorescence microscopy and differential interference contrast optics. Images were collected with a digital Axiocam (Carl Zeiss).

\section{Bacterial challenges}

Adult LL01 and YR2 aphids were injected with approximately 300 E. coli (DH5 a) in insect Schneider medium (Sigma-Aldrich) using a nanoinject II nanoinjector (Drummond Scientific Company) under a dissecting microscope.

To study changes in MIF gene expression of LL01 aphids during secondary symbiont infections, 12-days-old aphids 
from the lineages YR2-Ri, YR2-Hd or YR2-Ss were pricked in the abdomen to collect hemolymph in a total volume of $200 \mu \mathrm{L}$ aphid Schneider medium (Sigma-Aldrich). Pelleted secondary symbionts were resuspended in $50 \mu \mathrm{L}$ of insect Schneider medium and counted under a microscope, using a counting chamber. Approximately 300 facultative secondary symbionts were injected in each adult LL01 aphid using a nanoinject II nanoinjector (Drummond Scientific Company) under a dissecting microscope. Control aphids were injected with sterile Schneider medium. Injected aphids were either frozen at $-80^{\circ} \mathrm{C}$ at one and 4 days post-injection, or allowed to deposit nymphs for 2 days. 12-days-old adults from the first generation (F1) of injected aphids were collected and stored at $-80^{\circ} \mathrm{C}$ until RNA extraction.

\section{Production of aphids parasitized by Aphidius ervi}

Three days old aphid nymphs from LL01, YR2, YR2-Ri, YR2-Hd or YR2-Ss were individually exposed to a single Aphidius ervi female wasp for $24 \mathrm{hr}$. Aphids were collected 3 days after parasitoid exposure and stored at $-80^{\circ} \mathrm{C}$ until RNA extraction. For each sample, a set of aphids was maintained on plants under normal conditions to observe mummification and validate the success of parasitoid infection.

\section{Real-time quantitative reverse transcriptase-PCR (RT-qPCR)}

Total RNA was isolated from homogenized tissues (whole bodies or hemocytes) using the RNeasy micro plus kit (Qiagen) and quantified with a nanodrop (Agilent). Firststrand cDNA was generated from 500 ng RNA using iScript cDNA synthesis kit, according to standard procedures (Biorad, California, USA). Gene specific primers were designed with Primer3 software [50]. Real-time quantitative PCR was performed on a DNA Engine 2 (MJ Research, Minnesota, USA) with qPCR MasterMix Plus for SYBR green I (Eurogentec, Belgium) using one internal reference transcript (elongation factor 1- $\alpha$, NCBI RefSeq XM_001951252.2). Running parameters were $95^{\circ} \mathrm{C}$ for $10 \mathrm{~min}$, followed by 40 cycles of $95^{\circ} \mathrm{C}$ for $30 \mathrm{~s}, 60^{\circ} \mathrm{C}$ for $30 \mathrm{~s}, 68^{\circ} \mathrm{C}$ for $30 \mathrm{~s}$. All amplifications were performed in triplicate assays. Signal intensity was measured at the end of each elongation phase and results were analyzed using the Opticon 3.1 software provided by MJ Research. To assess the specificity of the PCR amplification, a melting curve analysis of the amplicon was performed at the end of each reaction and a single peak was always observed. Standard curves were established with four serial dilutions of first-strand cDNAs, ranging from $1 / 10$ to $1 / 10000$. ApMIFs expression levels were normalized to expression of the internal control elongation factor 1- $\alpha$ (EF1) using the comparative CT method (Applied biosystems, USA).

\section{Statistical analyses}

All experiments were repeated three times independently, and each sample corresponds to pools of 10-15 individuals. Differences in relative ApMIFs gene expression levels were tested for statistical significance by oneway ANOVA and the tukey-Kramer test on row data (Software Prism v.5.0, GraphPad). Expression of the target genes are presented either as an expression ratio normalized to EF1 expression or as a relative expression normalized to EF1 expression and to expression in the control sample.

\section{Availability of supporting data}

The data set supporting the phylogenetic analysis is available in the TreeBase repository (http://purl.org/phylo/tree base/phylows/study/TB2:S16212).

\section{Additional file}

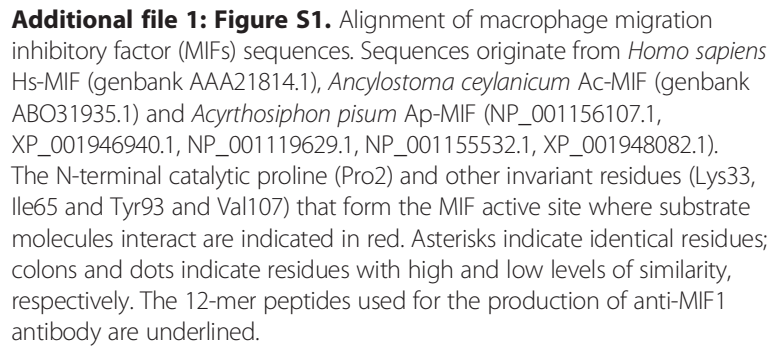

Additional file 1: Figure S1. Alignment of macrophage migration inhibitory factor (MIFs) sequences. Sequences originate from Homo sapiens Hs-MIF (genbank AAA21814.1), Ancylostoma ceylanicum Ac-MIF (genbank ABO31935.1) and Acyrthosiphon pisum Ap-MIF (NP_001156107.1, XP_001946940.1, NP 001119629.1, NP 001155532.1, XP 001948082.1). The N-terminal catalytic proline (Pro2) and other invariant residues (Lys33, Ile65 and Tyr93 and Val107) that form the MIF active site where substrate molecules interact are indicated in red. Asterisks indicate identical residues; colons and dots indicate residues with high and low levels of similarity, respectively. The 12-mer peptides used for the production of anti-MIF1 antibody are underlined.

\section{Competing interests}

The authors declare that they have no competing interests.

\section{Authors' contributions}

GD participated in the study design, carried out the molecular and expression studies and drafted the manuscript. ED carried out the database searches, sequence analyses and phylogenetic reconstructions. DC participated in the immunolabelling studies. JM.S provided the aphid clones and helped draft the manuscript. CC conceived the study, performed its design and coordination and helped to draft the manuscript. All authors read and approved the final manuscript.

\section{Acknowledgements}

This work has been supported by INRA and CNRS. Authors are grateful to T. Fukatsu, R. Koga and T. Tsuchida for providing YR2 lines with different secondary symbionts, and to Jean-Luc Gatti for help with the production of pre-immune serum.

\section{Author details}

${ }^{1}$ Sophia Agrobiotech Institute, INRA-CNRS-UNS, UMR 7254, 400 Route des Chappes, 06903 Sophia Antipolis, France. ${ }^{2}$ Current address: Institut de Recherche sur la Biologie de I'Insecte, UMR 7261, CNRS/Université François-Rabelais, Parc Grandmont, 37200 Tours, France. ${ }^{3}$ Institute of Genetics, Environment and Plant Protection, INRA, UMR 1349, Domaine de la Motte, BP 35327, 35653 Le Rheu Cedex, France.

Received: 24 April 2014 Accepted: 18 August 2014

Published: 5 September 2014

\section{References}

1. Hoffmann JA, Reichhart JM: Drosophila innate immunity: an evolutionary perspective. Nat Immunol 2002, 3(2):121-126. 
2. Flajnik MF, Du Pasquier L: Evolution of innate and adaptive immunity: can we draw a line? Trends Immunol 2004, 25(12):640-644.

3. Schulenburg H, Boehnisch C, Michiels NK: How do invertebrates generate a highly specific innate immune response? Mol Immunol 2007 44(13):3338-3344.

4. Dishaw LJ, Litman GW: Invertebrate allorecognition: the origins of histocompatibility. Curr Biol 2009, 19(7):R286-R288.

5. Baeza Garcia A, Pierce RJ, Gourbal B, Werkmeister E, Colinet D, Reichhart JM, Dissous C, Coustau C: Involvement of the cytokine MIF in the snail host immune response to the parasite Schistosoma mansoni. PLOS Pathog 2010, 6(9):e1001115.

6. IAGC: Genome sequence of the pea aphid Acyrthosiphon pisum. PLoS Biol 2010, 8(2):e1000313

7. Elsik CG: The pea aphid genome sequence brings theories of insect defense into question. Genome Biol 2010, 11(2):106.

8. Gerardo NM, Altincicek B, Anselme C, Atamian H, Barribeau SM, de Vos M, Duncan EJ, Evans JD, Gabaldon T, Ghanim M, Heddi A, Kaloshian I, Latorre A, Moya A, Nakabachi A, Parker BJ, Perez-Brocal V, Pignatelli M, Rahbe Y, Ramsey JS, Spragg CJ, Tamanes J, Tamarit D, Tamborindeguy C, Vincent-Monegat C, Vilcinskas A: Immunity and other defenses in pea aphids. Acyrthosiphon pisum. Genome Biol 2010, 11(2):R21.

9. Moran N, Baumann P: Phylogenetics of cytoplasmically inherited microorganisms of arthropods. Trends Ecol Evol 1994, 9(1):15-20.

10. Wilson AC, Ashton PD, Calevro F, Charles H, Colella S, Febvay G, Jander G, Kushlan PF, Macdonald SJ, Schwartz JF, Thomas GH, Douglas AE: Genomic insight into the amino acid relations of the pea aphid, Acyrthosiphon pisum, with its symbiotic bacterium Buchnera aphidicola. Insect Mol Biol 2010, 19(Suppl 2):249-258.

11. Oliver KM, Degnan PH, Burke GR, Moran NA: Facultative symbionts in aphids and the horizontal transfer of ecologically important traits. Annu Rev Entomol 2010, 55:247-266.

12. Schmitz A, Anselme C, Ravallec M, Rebuf C, Simon JC, Gatti JL, Poirie M: The cellular immune response of the pea aphid to foreign intrusion and symbiotic challenge. PLoS One 2012, 7(7):e42114.

13. Tsuchida T, Koga R, Horikawa M, Tsunoda T, Maoka T, Matsumoto S, Simon JC, Fukatsu T: Symbiotic bacterium modifies aphid body color. Science 2010, 330(6007):1102-1104.

14. Simon JC, Boutin S, Tsuchida T, Koga R, Le Gallic JF, Frantz A, Outreman Y, Fukatsu T: Facultative symbiont infections affect aphid reproduction. PLoS One 2011, 6(7):e21831.

15. Montllor CB, Maxmen A, Purcell AH: Facultative bacterial endosymbionts benefit pea aphids Acyrthosiphon pisum under heat stress. Ecological Entomology 2002, 27:189-195.

16. Oliver KM, Russell JA, Moran NA, Hunter MS: Facultative bacterial symbionts in aphids confer resistance to parasitic wasps. Proc Natl Acad Sci U S A 2003, 100(4):1803-1807.

17. Guay JF, Boudreault S, Michaud D, Cloutier C: Impact of environmental stress on aphid clonal resistance to parasitoids: Role of Hamiltonella defensa bacterial symbiosis in association with a new facultative symbiont of the pea aphid. J Insect Physiol 2009, 55(10):919-926.

18. Scarborough $\mathrm{CL}$, Ferrari J, Godfray HC: Aphid protected from pathogen by endosymbiont. Science 2005, 310(5755):1781.

19. Login FH, Balmand S, Vallier A, Vincent-Monegat C, Vigneron A, Weiss-Gayet M, Rochat D, Heddi A: Antimicrobial peptides keep insect endosymbionts under control. Science 2011, 334(6054):362-365

20. Poirie M, Coustau C: The evolutionary ecology of aphids' immunity. Isj-Invertebrate Survival Journal 2011, 8(2):247-255.

21. Ratzka C, Forster F, Liang C, Kupper M, Dandekar T, Feldhaar H, Gross R: Molecular characterization of antimicrobial peptide genes of the carpenter ant Camponotus floridanus. PLoS One 2012, 7(8):e43036.

22. Calandra T, Roger T: Macrophage migration inhibitory factor: a regulator of innate immunity. Nat Rev Immunol 2003, 3(10):791-800.

23. Merk M, Mitchell RA, Endres S, Bucala R: D-dopachrome tautomerase (D-DT or MIF-2): doubling the MIF cytokine family. Cytokine 2012, 59(1):10-17.

24. Wang BZ, Zhang ZP, Wang YL, Zou ZH, Wang GD, Wang SH, Jia XW, Lin P: Molecular cloning and characterization of macrophage migration inhibitory factor from small abalone Haliotis diversicolor supertexta. Fish \& Shellfish Immunology 2009, 27(1):57-64.

25. Parisi MG, Toubiana M, Mangano V, Parrinello N, Cammarata M, Roch P: MIF from mussel: coding sequence, phylogeny, polymorphism, 3D model and regulation of expression. Dev Comp Immunol 2012, 36(4):688-696.
26. Maizels RM, Balic A, Gomez-Escobar N, Nair M, Taylor MD, Allen JE: Helminth parasites - masters of regulation. Immunol Rev 2004, 201:89-116.

27. Augustijn KD, Kleemann R, Thompson J, Kooistra T, Crawford CE, Reece SE, Pain A, Siebum AH, Janse CJ, Waters AP: Functional characterization of the Plasmodium falciparum and $P$. berghei homologues of macrophage migration inhibitory factor. Infect Immun 2007, 75(3):1116-1128.

28. Vermeire JJ, Cho Y, Lolis E, Bucala R, Cappello M: Orthologs of macrophage migration inhibitory factor from parasitic nematodes. Trends Parasitol 2008, 24(8):355-363.

29. Bowen CJ, Jaworski DC, Wasala NB, Coons LB: Macrophage migration inhibitory factor expression and protein localization in Amblyomma americanum (Ixodidae). Exp Appl Acarol 2010, 50(4):343-352.

30. Altschul SF, Gish W, Miller W, Myers EW, Lipman DJ: Basic local alignment search tool. J Mol Biol 1990, 215(3):403-410.

31. Cho $Y$, Jones BF, Vermeire JJ, Leng L, DiFedele L, Harrison LM, Xiong $H$, Kwong YK, Chen Y, Bucala R, Lolis E, Cappello M: Structural and functional characterization of a secreted hookworm Macrophage Migration Inhibitory Factor (MIF) that interacts with the human MIF receptor CD74. $\mathrm{J}$ Biol Chem 2007, 282(32):23447-23456.

32. Kleemann R, Rorsman H, Rosengren E, Mischke R, Mai NT, Bernhagen J: Dissection of the enzymatic and immunologic functions of macrophage migration inhibitory factor. Full immunologic activity of $\mathrm{N}$-terminally truncated mutants. Eur J Biochem 2000, 267(24):7183-7193.

33. Dobson SE, Augustijn KD, Brannigan JA, Schnick C, Janse CJ, Dodson EJ, Waters AP, Wilkinson AJ: The crystal structures of macrophage migration inhibitory factor from Plasmodium falciparum and Plasmodium berghei. Protein Sci 2009, 18(12):2578-2591.

34. Flieger O, Engling A, Bucala R, Lue H, Nickel W, Bernhagen J: Regulated secretion of macrophage migration inhibitory factor is mediated by a non-classical pathway involving an ABC transporter. FEBS Lett 2003, 551(1-3):78-86

35. Petersen TN, Brunak S, von Heijne G, Nielsen H: SignalP 4.0: discriminating signal peptides from transmembrane regions. Nat Methods 2005, 8(10):785-786.

36. Bendtsen JD, Kiemer L, Fausboll A, Brunak S: Non-classical protein secretion in bacteria. BMC Microbiol 2005, 5:58.

37. Rahbe Y, Febvay G: Protein Toxicity to Aphids - An invitro test on Acyrthosiphon pisum. Entomologia Experimentalis Et Applicata 1993, 67(2):149-160.

38. Henry LM, Roitberg BD, Gillespie DR: Host-range evolution in Aphidius parasitoids: fidelity, virulence and fitness trade-offs on an ancestral host. Evolution 2008, 62(3):689-699.

39. Chen $D Q$, Purcell $A H$ : Occurrence and transmission of facultative endosymbionts in aphids. Curr Microbiol 1997, 34(4):220-225.

40. Fukatsu T, Tsuchida T, Nikoh N, Koga R: Spiroplasma symbiont of the pea aphid, Acyrthosiphon pisum (Insecta: Homoptera). Appl Environ Microbio 2001, 67(3):1284-1291

41. Bacher M, Metz CN, Calandra T, Mayer K, Chesney J, Lohoff M, Gemsa D, Donnelly T, Bucala R: An essential regulatory role for macrophage migration inhibitory factor in T-cell activation. Proc Natl Acad Sci U S A 1996, 93(15):7849-7854.

42. Renner $\mathrm{P}$, Roger $\mathrm{T}$, Calandra $\mathrm{T}$ : Macrophage migration inhibitory factor: gene polymorphisms and susceptibility to inflammatory diseases. Clin Infect Dis 2005, 41(Suppl 7):S513-S519.

43. Rosado Jde D, Rodriguez-Sosa M: Macrophage migration inhibitory factor (MIF): a key player in protozoan infections. Int J Biol Sci 2011, 7(9):1239-1256.

44. Das R, Koo MS, Kim BH, Jacob ST, Subbian S, Yao J, Leng L, Levy R, Murchison C, Burman WJ, Moore CC, Scheld WM, David JR, Kaplan G, MacMicking JD, Bucala R: Macrophage migration inhibitory factor (MIF) is a critical mediator of the innate immune response to Mycobacterium tuberculosis. Proc Natl Acad Sci U S A 2013, 110(32):E2997-E3006.

45. Vigneron A, Charif D, Vincent-Monégat C, Vallier A, Gavory F, Wincker $P$, Heddi A: Host gene response to endosymbiont and pathogen in the cereal weevil Sitophilus oryzae. BMC Microbiol 2012, 12(1):S14.

46. Pruitt KD, Tatusova T, Brown GR, Maglott DR: NCBI Reference Sequences (RefSeq): current status, new features and genome annotation policy. Nucleic Acids Res 2002, 40(Database issue):D130-D135.

47. Edgar RC: MUSCLE: multiple sequence alignment with high accuracy and high throughput. Nucleic Acids Res 2004, 32(5):1792-1797. 
48. Guindon S, Dufayard JF, Lefort V, Anisimova M, Hordijk W, Gascuel O: New algorithms and methods to estimate maximum-likelihood phylogenies: assessing the performance of PhyML 3.0. Syst Biol 2010, 59(3):307-321.

49. Cortes T, Tagu D, Simon JC, Moya A, Martinez-Torres D: Sex versus parthenogenesis: a transcriptomic approach of photoperiod response in the model aphid Acyrthosiphon pisum (Hemiptera: Aphididae). Gene 2008, 408(1-2):146-156.

50. Rozen S, Skaletsky H: Primer3 on the WWW for general users and for biologist programmers. Methods Mol Biol 2000, 132:365-386.

doi:10.1186/1471-2164-15-762

Cite this article as: Dubreuil et al.: Diversification of MIF immune

regulators in aphids: link with agonistic and antagonistic interactions. BMC Genomics 2014 15:762.

\section{Submit your next manuscript to BioMed Central and take full advantage of:}

- Convenient online submission

- Thorough peer review

- No space constraints or color figure charges

- Immediate publication on acceptance

- Inclusion in PubMed, CAS, Scopus and Google Scholar

- Research which is freely available for redistribution 